\title{
Paraplegia in a patient with Von Hippel Lindau syndrome: surgical and reconstructive treatment of Marjolin's ulcer. A case report
}

\author{
A Scalise ${ }^{1}$, C Tartaglione ${ }^{1}$, M Pierangeli ${ }^{1}$, E Bolletta $^{1}$, M Fraccalvieri ${ }^{2}$, L Grassetti ${ }^{1}$, M Ottonello $^{3}$, \\ G Nicoletti $^{4}$, A Massone ${ }^{3}$ and G Di Benedetto ${ }^{1}$
}

Study design: Marjolin's ulcer is a squamous cell carcinoma that develops in posttraumatic scars and chronic wounds. Suspicion of such lesions should be raised in chronic wounds demonstrating characteristic changes. We have reported the peculiar phenomenon of malignant transformation of chronic pressure sores that occurred in a paraplegic patient.

Objectives: The aim of this study was to cover the extensive defects by a last resort reconstructive option.

Setting: Department of Plastic and Reconstructive Surgery, Università Politecnica delle Marche, Ancona, Italy.

Methods and results: A 40-year-old paraplegic man, with multiple hemangioblastomas of the brain and spinal cord due to Von Hippel Lindau syndrome developed pressure ulcers with unstable healing over the sacral, trochanteric, bilateral, and ischiatic areas after 15 years from neurosurgery. The biopsy result showed an invasive squamous carcinoma. Carcinomas in pressure sores are highly aggressive, and they need to be treated more radically. In our case we opted for a demolitive surgical treatment including musculocutaneous rotational flap harvested from total left thigh to cover the extensive defects. The limb was previously disarticulated. Conclusion: In Marjolin's ulcer, multiple biopsies are the first-line modality for the early diagnosis as they are a safe method with high rate of accuracy. First-line treatment is surgery consisting of radical excision with lymph node dissection, if they are involved. Adjuvant radiation therapy may be used in selected patients. Management of massive pelvic defects can be a challenging problem. The pedicled lower limb flap offers a technique that can be considered as a last resort procedure for extensive defects where other options are insufficient or not available anymore. In our case the patient is disease-free after 2 years of follow-up.

Spinal Cord (2014) 52, S1-S3; doi:10.1038/sc.2014.111

\section{INTRODUCTION}

Pressure sores remain a common problem in bedridden patients, 25$85 \%$ incidence. $^{1}$ Chronic wounds and scar tissues are prone to an increased risk of skin cancer, known as Marjolin's ulcer. The commonest type of carcinoma arising from these lesions is squamous cell carcinoma with a bad prognosis. ${ }^{2}$

\section{CASE REPORT}

Marjolin's ulcer was observed in a 40-year-old patient with Von Hippel Lindau syndrome leading to permanent paraplegia due to hemangioblastomas.

At 25 years of age he developed a gait ataxia. A contrast-enhanced magnetic resonance imaging (MRI) scan of the brain and spinal cord evidenced lesions within the posteroinferior portion of the fourth ventricle and the second, third, sixth and twelfth dorsal segments, with hemangioblastoma characters. Ventricular lesion was removed and an improvement of the gait disturbance was achieved. After 1 year, he developed an acute paraplegia with sensitive T9 level and loss of sphincteric control. A contrast-enhanced MRI disclosed an increase in volume of the intramedullary lesion at T10 with cord swelling. The patient was treated in emergency by a T9-T11 decompressive laminectomy followed by the tumor removal.

Postoperative MRI did not show complications.

Nonetheless, he never experienced a neurological recovery from the admission deficit. He developed multiple, unstable healing ulcers after 2 years.

In January 2012 the patient was admitted in our Department.

He presented lesions over the left ischiatic, trochanteric, bilateral and sacral areas (III/IV grade, National Pressure Ulcer Advisory Panel) and fistula abscess in the posterior region of the left thigh (Figure 1).

Malignant degeneration features were noticed on the ischiatic ulcer and biopsies were performed.

The histological examination identified a squamous cell carcinoma only on the left ischiatic ulcer.

The contrast-enhanced computed tomography (CT) and MRI scans did not show any systemic involvement.

The complicating factors included severe malnutrition, poor physical state and infection by a multiresistant germ (Pseudomonas aeruginosa). A specific antibiotic therapy with ciprofloxacin was started.

${ }^{1}$ Department of Plastic and Reconstructive Surgery, Università Politecnica delle Marche, Ancona, Italy; ${ }^{2}$ Department of Plastic and Reconstructive Surgery, Università di Torino (ASO, Città della Salute e della Scienza), Torino, Italy; ${ }^{3}$ Spinal Unit, Azienda Ospedaliera Santa Corona, Pietra Ligure, Italy and ${ }^{4}$ Department of Clinical Surgical, Diagnostic and Paediatric Sciences, Università di Pavia, Italy

Correspondence: Professor A Scalise, Department of Plastic and Reconstructive Surgery, Università Politecnica delle Marche, Via Conca n. 71, Torrette, Ancona 60126, Italy. E-mail: a.scalise@univpm.it

Received 14 March 2014; revised 20 May 2014; accepted 28 May 2014 

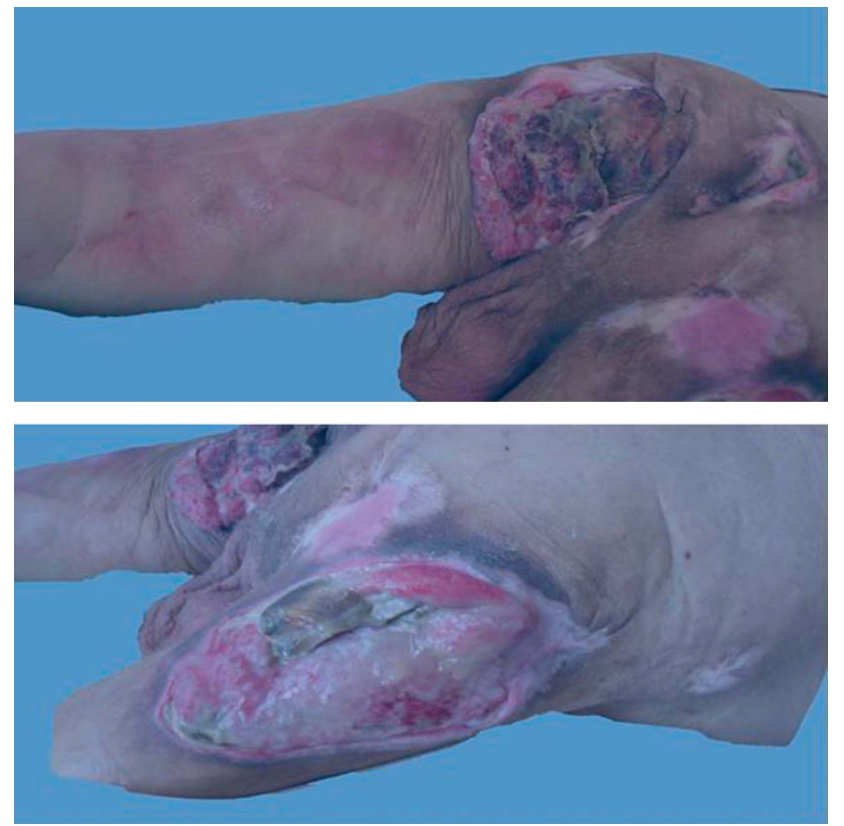

Figure 1 Marjolin's ulcer over the left ischiatic region, trochanteric, bilateral and sacral areas (III/IV grade, National Pressure Ulcer Advisory Panel) and fistula abscess in the posterior region of the left thigh.

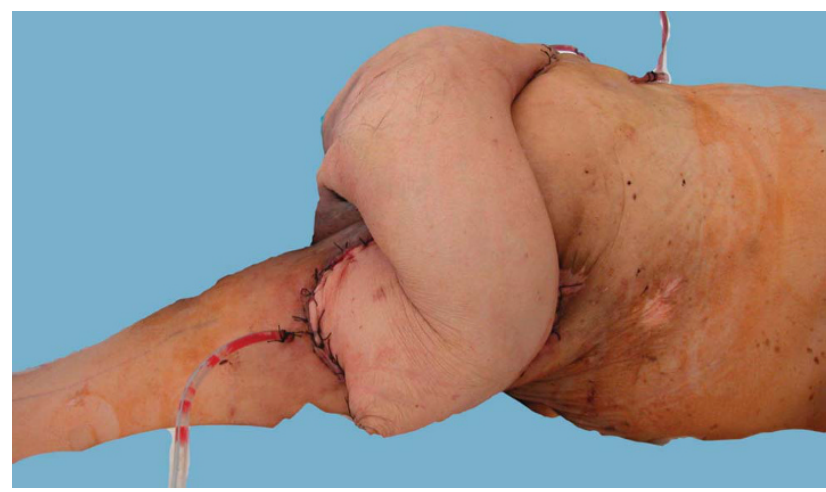

Figure 2 Postoperative result. A musculocutaneous rotational flap from the left thigh (anterior, medial and lateral regions) until the proximal third of the leg covered the ischiatic left ulcer and sacral one.

A defunctioning colostomy was performed to minimize the fecal contamination.

We opted for a radical surgical treatment.

The lesions were radically excised until bone (with oncological radicality). A bone biopsy was sent for a bone culture to guide the antibiotic therapy for osteomyelitis (the examination of bone culture resulted negative).

We previously disarticulated the flap.

An incision was made along the posterior aspect of the left thigh, identifying the lateral intermuscular septum.

This incision was continued beyond the popliteal fossa until tibial peroneal trunk. The periosteum over the femur was raised and the flap was raised off the entire length of the femur.

The articular capsule was divided at its attachment to the pelvis and division of the ligamentum teres completed the disarticulation.

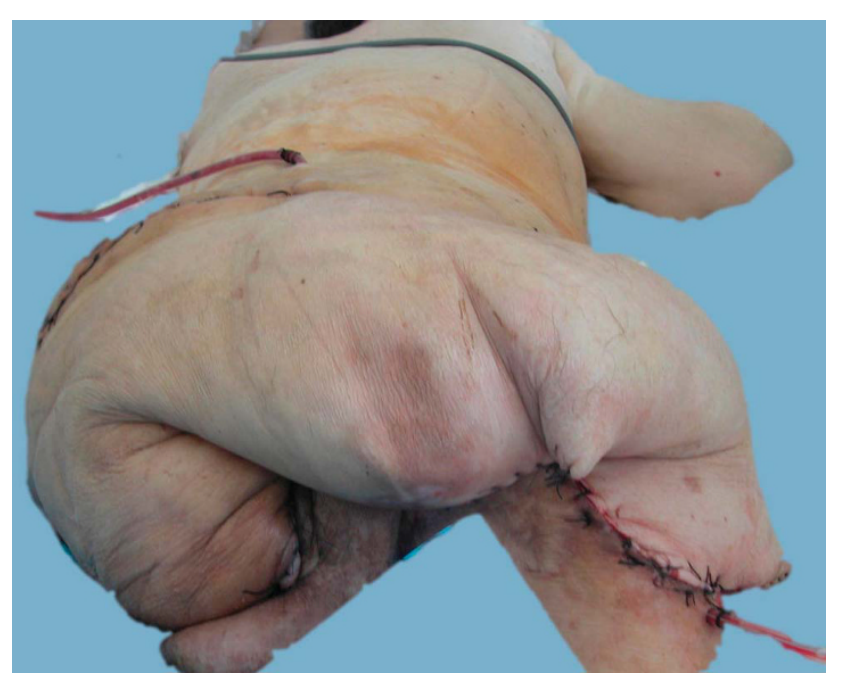

Figure 3 Post-operative result. A tubularized flap extension covered the contralateral trochanteric defect.

The periosteal and muscular elevation was performed to close the acetabular cavity.

The total thigh flap (TTF) was turned on itself, and the deep muscles sutured into place.

We preferred to use a musculocutaneous rotational flap from the left thigh (anterior, medial and lateral regions) until the proximal third of the leg to cover the ischiatic left ulcer and sacral area. A tubularized flap extension covered the contralateral trochanteric defect (Figures 2 and 3).

After 2 months of air-fluidized bed, the patient was discharged to the rehabilitation department.

The patient is disease-free yet (24 months of follow-up), CT scan and MRI with contrast medium did not show any systemic and local involvement.

\section{DISCUSSION}

Pressure sores are one of the most common secondary conditions in paraplegic patients.

The incidence of chronic non-healing ulcers undergoing malignant transformation is a rare phenomenon, $0.5 \%$ incidence. $^{3}$

Awareness of the malignant potential may help decrease the morbidity.

In the clinical history, it is important to analyze the morphological changes through the most appropriate diagnostic techniques, as in the advanced stages even an aggressive surgical procedure may prove inadequate.

First-line treatment consists of radical excision with a range of $2 \mathrm{~cm}$ from the margin of the ulcer and potential lymph node dissection (according to fine-needle aspiration cytology and CT scan).

Adjuvant radiation therapy may be used; however, the response to systemic chemotherapy is generally poor. ${ }^{4}$ In our case, according to radiotherapist we decided not to perform radiotherapy. Management of massive pelvic defects can be a challenging problem. The pedicled lower limb flap offers a last resort technique for extensive defects where other options are insufficient or not available anymore.

The TTF is a well-vascularized myocutaneous flap and provides valuable viable tissue, improving the status of the paraplegic patient with multiple pressure ulcers. ${ }^{5}$

We demonstrated the necessity of rigorous surgical treatment because the clinical course of Marjolin's ulcer is rapid and fulminant. 
Patients should be followed up for the rest of their life.

As a result of 24-month follow-up, our patient is disease-free.

\section{CONFLICT OF INTEREST}

The authors declare no conflict of interest.

\section{ACKNOWLEDGEMENTS}

AS, GDB and LG led the study design, coordination and had the role of first surgeons; MP, MF, MO and AM collected the clinical data before and after surgery; CT and EB participated in the study design and drafted the manuscript. All authors read and approved the final manuscript.
1 Biglari b, Buchler A, Reitzel T, Swing T, Gerner HJ, Ferbert T et al. A retrospective study on flap complications after pressure ulcer surgery in spinal cord-injured patients. Spinal Cord 2014; 52: 80-83.

2 Yu N, Long X, Lujan-Hernandez JR, Hassan KZ, Bai M, Wang Y et al. Marjolin's ulcer: a preventable malignancy arising from scars. World J Surg Oncol 2013; 11: 313 .

3 Htwe O, Leow M, Naicker AS. Marjolin's Ulcer complicating a pressure sore. Eur J Gen Med 2011; 8: 246-249.

4 Choi JY, Bae YC, Nam SB, Bae SH. Impact of disturbed wound healing after surgery on the prognosis of Marjolin's Ulcer. Arch Plast Surg 2013; 40: 198-202.

5 Grassetti L, Scalise A, Lazzeri D, Carle F, Agostini T, Gesuita R et al. Perforator flaps in late-stage pressure sores treatment: outcome analysis of 11-year-long experience with 143 patients. Ann Plast Surg 2013 (e-pub ahead of print 12 June 2013). 